\title{
Immunopathology of COVID-19 caused by SARS-CoV-2: A brief review
}

\author{
Bramhadev Pattnaik $^{1 *}$, Pinaki Panigrahi ${ }^{2}$ and Mahendra P Yadav ${ }^{\mathbf{3}}$ \\ ${ }^{1}$ One Health Center for Surveillance and Disease Dynamics, AIPH University, \\ Bhubaneswar, Odisha and Former Director, ICAR-Directorate of Foot and Mouth \\ Disease, Mukteshwar, India \\ ${ }^{2}$ Department of Pediatrics, Division of Neonatal-Perinatal Medicine, Georgetown \\ University Medical Center, Washington, DC, USA \\ ${ }^{3}$ Former Vice-Chancellor, SVP University of Agriculture and Technology, Meerut, India \\ *Corresponding Author: Bramhadev Pattnaik, One Health Center for Surveillance \\ and Disease Dynamics, AIPH University, Bhubaneswar, Odisha and Former Director, \\ ICAR-Directorate of Foot and Mouth Disease, Mukteshwar, India.
}

Received: May 22, 2020

Published: June 29, 2020

(C) All rights are reserved by Bramhadev

Pattnaik, et al.

\begin{abstract}
The novel coronavirus SARS-CoV-2 responsible for Coronavirus infectious disease of 2019 (COVID-19) in human, now a Global pandemic affecting 212 countries in all the five Continents, damages the cells that have ACE2 receptor expression on their surface. Hoffman., et al. (2020) observed that in addition to ACE2 receptor required for cellular attachment of the virion, cellular entry mechanism of the virus requires a cellular serine protease, TMPRSS2 and because alveolar type 2 cells express higher levels of both ACE2 and TMPRSS2 receptors, these cells might be the primary entry point for the virus in the lung. Intestine and kidney also have high expression of ACE2. The virus along with the ACE2 receptor enters the cells by endocytosis that results in reduction of ACE2 on cells, and as a consequence serum level of angiotensin II increases. Angiotensin II acts both as a vasoconstrictor and pro-inflammatory cytokine. Exposure to the virus does not necessarily cause infection, and not all people infected develop ARDS (acute respiratory distress syndrome) or succumb to the disease. Though people of all age group and sex are susceptible, the disease could be more fatal in elderly persons > 60 years of age. Extensive lung damage due to elicitation of Cytokine Storm (cytokine release syndrome; CRS) has been described by Hirano and Murakami (2020) and Shi., et al (2020). Role of specific HLA loci and alleles (class I or II) in developing protective immunity to this virus infection remains to be elucidated. It has been reported by Iwasaki and Yang (2020) that antibodies to SARS-CoV-2 at low concentrations as well as low affinity antibodies in the body can result in antibody-dependent enhancement (ADE) by utilizing Fc receptors on immune cells including macrophages, monocytes and B lymphocytes. ADE has detrimental effect in some patients with virus specific antibodies. High WBC count with associated lymphocytopenia is common in the COVID-19 patients. Ganji., et al. (2020) have reported significant reduction in the numbers of circulating lymphocytes and platelets, $\mathrm{CD} 4^{+}$: CD8 ${ }^{+}$ratio of $2: 1$, and higher expression of $\mathrm{CD}^{+}$and hyperactivation of CTLs and no significant change in the expression level of CD4 ${ }^{+}$compared to healthy individuals. Anti-viral immune response to SARS-CoV-2 infection was due to over expression of CD8 and hyper activation of CTLs. Pathophysiology and pathology of the disease has been elaborated by Yuki., et al. (2020) and Sahu., et al (2020). The present review compiles the aspects of pathogenesis and involvement of the host immune system in aggravating the disease through the process of immune response.
\end{abstract}

Keywords: Coronavirus; COVID-19; Immunopathology; Immune Response; Pandemic; SARS

\section{Introduction}

The coronaviruses were first discovered in the 1930s with the isolation of infectious bronchitis virus (IBV) that causes an acute respiratory infection in domesticated chickens. The Human coronaviruses were discovered in the 1960s. A pneumonia outbreak in human caused by a novel strain of coronavirus (nCoV) was diagnosed in Wuhan, China, in December 2019. The virus was given the interim name 2019-nCoV by the World Health Organization (WHO) and later renamed as Severe Acute Respiratory Syndrome (SARS)-CoV-2 by the International Committee on Taxonomy of Viruses_(ICTV). It has been identified as a new strain of group 2B 
Beta-coronavirus, with nucleotide identity of $96 \%, 91 \%, 80 \%, 55 \%$ and $50 \%$ with coronaviruses of bat, pangolin, SARS, Middle East Respiratory Syndrome (MERS) and common cold, respectively [8]. The sequence identity in the $S$ (spike glycoprotein) gene of SARSCoV-2 with the bat origin viruses was estimated to be only $75 \%$; the $\mathrm{S}$ protein determines host tropism and transmissibility of the virus. There is less genetic similarity of SARS-CoV-2 with SARSCoV of 2003 and MERS-CoV of 2012 [9]. The receptor binding domain (RBD) of both SARS-CoV-2 of 2019 and SARS-CoV of 2003 recognize and bind to angiotensin converting enzyme 2 (ACE 2) receptor on the susceptible cells, whereas that of MERS-CoV binds to DPP4 (dipeptidyl peptidase 4) receptor [2,7]. On January 30 , the WHO declared the novel coronavirus outbreak (COVID-19) a Global Public Health Emergency, and more than 200 countries, and all the continents, are now affected. In India the case numbers as well as death rate are much less than many other countries. People of all ages and both the sexes are susceptible to COVID-19. Older people are at higher risk of dying from the disease as compared to younger ones. The fatality rate is about $3.6 \%$ in people of $>60$ years of age and about $0.2 \%$ in younger ones of $<40$ years old [7]. The disease has three stages, viz. asymptomatic with or without detectable virus, symptomatic but not severe with detectable virus and severe respiratory disease (acute respiratory distress syndrome; ARDS) with high virus load in the body $[6,10]$. Cytokine release storm/syndrome (CRS) and antibody dependent enhancement (ADE) have been detected to complicate the pathology. Both innate and adaptive immunity play role in defending the host species from microbial infection, and genetic difference due to HLA variability results in differences in immune response to the invading pathogen. However, the specific HLA loci and the alleles (class I or II) associated with induction of protective antibodies and immunity to SARS-CoV-2 remains to be identified [3]. The present review includes some of the important findings and observations made by different groups of scientists investigating the disease in the areas of virus-host cell interaction and immunopathology responsible for ARDS.

Currently, medical treatment is largely supportive/symptomatic and drugs including lopinavir-ritonavir, remdesivir, hydroxychloroquine and azithromycin have been tested in clinical trials $[11,12]$. But none of these are specific for the causative virus. Hydroxychloroquine is being used in many countries for treating patients. Lacking an effective antiviral drug or vaccine, affected countries have resorted to social distancing by implementing lockdowns to check human to human and further community spread of the virus.
Causative agent of COVID-19 and other human coronaviruses

Coronaviruses (Order Nidovirales and family Coronaviridae) are enveloped, positive-sense, single-stranded RNA viruses of $\sim 30$ $\mathrm{kb}$ that can infect a wide variety of host species. Of the four genera $(\alpha, \beta, \gamma$, and $\delta$ ) of Coronaviridae, genus $\alpha$ and $\beta$ coronaviruses infect only mammals [13]. A total of seven CoVs have been reported causing mild to severe disease in humans; 04 with seasonal circulation causing mild cold (HKU1, NL63, OC43 and 229E) and the remaining 03 are more pathogenic and zoonotic ones, viz., SARS-CoV (2003), MERS-CoV (2012) and SARS-CoV-2 (2019), that originated in different bat species and transmitted to human through an intermediate host [Civet in case of SARS-CoV, Dromedary Camel in case of MERS-CoV, and possibly Pangolin [14] in case of SARS-CoV-2 causing COVID-19. Human coronaviruses responsible for common cold belong to genus $\alpha$ coronavirus, whereas SARS-CoV, MERS- CoV and SARS-CoV-2 belong to genus $\beta$ coronavirus. The SARS-CoV- 2 belongs to the subgenus Sarbecovirus (lineage B). The genome organization of coronavirus is: $5^{\prime}$-leader-UTR-replicase/transcriptase (RdRp)-spike (S)-envelope (E)-membrane (M)-nucleocapsid (N)3'UTR-poly (A) tail. At least 12 coding regions have been predicted in the virus genome, and spike protein of SARS-CoV-2 is longer than SARS-CoV of 2003 and MERS-CoV of 2012 [9]. The life cycle of the virus in the host cell consists of 5 steps, namely, attachment, penetration, biosynthesis of non-structural and structural proteins, maturation and release of new virion particles. Coronaviruses consist of four structural proteins; Spike (S), membrane (M), envelop (E) and nucleocapsid (N).

\section{Virus receptor and cellular entry}

Spike glycoprotein (S) protruding from the viral surface is composed of a transmembrane trimetric glycoprotein, which determines the diversity of coronaviruses and host tropism. The spike comprises two functional subunits; S1 subunit is responsible for binding to the host cell receptor and S2 subunit for the fusion of the viral and cellular membranes for endocytosis [9]. The receptor binding domain (RBD) of beta-coronaviruses is located in the C- terminus of S1 domain. The RBD region of SARS-CoV-2 $(29,903$ bases) spans from nucleotide 1030-1651 [15]. The RBD has a receptor binding motif (RBM) composed of 70 amino acids, the blockade of which results in neutralization of the SARS-CoV and there is only $59 \%$ similarity in amino acid sequence between the RBM of SARS-CoV and SARS-CoV-2, as against $82 \%$ amino acid similarity in the entire RBD region, and 94\% similarity outside the RBM region [16]. Angiotensin converting enzyme 2 (ACE2) was identified as a functional receptor for SARS-CoV [17]. Structural and functional analysis showed that the spike for SARS CoV-2 also binds to ACE2 
as its receptor [1,18-20]. Hoffman., et al. [1] further showed that ACE2 dependent virus entry required cellular transmembrane serine protease 2 (TMPRSS2). ACE2 expression is high in lung, heart, ileum, kidney and bladder [21]. In lung, ACE2 is highly expressed on the epithelial cells in the alveolar space. As alveolar type 2 cells highly express both ACE2 and TMPRSS2, these cells may be the primary entry point for the virus in the lung [2]. Hoffmann., et al. [1] demonstrated that the SARS-CoV-2 uses ACE2 for entry into cells, and TMPRSS2 and the endosomal cysteine proteases cathepsin B and $L(C a t B / L)$ for priming of spike protein. After binding of SARS$\mathrm{CoV}-2$ to the host protein, the spike protein undergoes protease cleavage at the S1/S2 junction for activation [22]. After the cleavage of the S1/S2 site, S1 and S2 subunits remain noncovalently bound and the distal S1 subunit contributes to the stabilization of the membrane anchored by the S2 subunit [20]. Subsequent cleavage at the $\mathrm{S} 2$ site presumably activates the spike for membrane fusion via irreversible conformational changes. The coronavirus spike is unusual among viruses because a range of different proteases can cleave and activate it [23]. The characteristics unique to SARS-CoV-2 among coronaviruses is the existence of furin cleavage site ("RPPA" sequence) at the S1/S2 site [23]. The S1/S2 site of SARS-CoV-2 is entirely subjected to cleavage during biosynthesis in a drastic contrast to SARS-CoV spike, which is incorporated into assembly without cleavage [20]. Though the S1/S2 site is sensitive to cleavage by proteases TMPRSS2 and cathepsin B/L, the ubiquitous expression of furin likely makes this virus very pathogenic $[1,22]$. As ACE2 is highly expressed on the apical side of lung epithelial cells in the alveolar space [24]; this virus can destroy these cells. This is in line with the fact that the early lung injury is often seen in the distal airway of SARS-CoV.

COVID-19: incubation period, clinical symptoms and epidemiology

The SARS-CoV-2 virus primarily affects the respiratory system, although other organ systems are also susceptible. Initially, COVID-19 cases were mostly observed among elderly people [25]. Subsequently, as the outbreak progressed, children of $<18$ years of age were also found affected with the disease. However, the incidence is much lower in children. Epidemiological studies have shown that mortality is higher in elder people as compared to younger ones $[11,18]$. Co-morbidity with cardiac ailment, diabetes and hypertension are considered to be risk factors for development of fatal ARDS. The average incubation period is of about 5 days (up to $\sim 14$ days) with average case-fatality rate of $2.3 \%$ [26,27]. Following Global spread of the disease, as on 04 May 2020 (05.02 GMT), the number of COVID-19 cases was the highest in the U.S.A., followed by (to name a few) Spain, Italy, U.K., France, Germany, Tukey, Iran,
Russia, China, Brazil, Canada, and Belgium (www.worldometers. info/coronavirus) with extrapolated case fatality rates (CFR; Total deaths/Total cases, \%) of 5.77, 10.22, 13.7, 15.2, 14.75, 4.14, 2.7, $6.37,0.95,5.6,6.9,6.2$ and 15.7 , respectively. During the same period, in India the CFR was about $3.27 \%$. This difference in CFR may be due to the age of the people infected with the virus; age could be directly proportional to the number of deaths, in addition to the extent of co-morbidity. The differences in CFR may also be due to the difference in total number of the people tested and cases diagnosed. HLA loci and alleles (either MHC class I or II) responsible for susceptibility/resistance to COVID-19 are yet to be identified.

According to WHO, symptomatic Covid-19 patients show most common symptoms like fever, tiredness, and dry cough. Some patients may have aches and pains, nasal congestion, runny nose, sore throat or diarrhoea. These symptoms are usually mild and begin gradually. Some people become infected but do not develop any symptoms or feel unwell. Most people (about 80\%) recover from the disease. Around 15\% people infected with SARS-CoV-2 become seriously ill with difficulty in breathing, and older people with co-morbidity are more likely to develop serious illness like ARDS needing ventilator support.

\section{Treatment options and disease management}

Currently, medical treatment is largely supportive/symptomatic, and drugs including lopinavir-ritonavir, remdesivir, hydroxychloroquine, and azithromycin have been tested in clinical trials [12] and are being used under compassionate ground. However, none of these are specific for the causative virus. Patients in a recent clinical trial in the U.S. receiving Remdesivir recovered faster than those getting placebo (https://www.niaid.nih.gov/newsevents/nih-clinical-trial-shows-remdesivir-accelerates-recoveryadvanced-covid-19). Remdesivir is an antiviral drug that has been used previously in Ebola virus infection in humans and has shown promise in animal models for treating Middle East respiratory syndrome (MERS) and severe acute respiratory syndrome (SARS), which are caused by other coronaviruses. Remdesivir is an adenosine nucleotide triphosphate analogue; its active metabolite interferes with the viral RNA-dependent RNA polymerase (RdRp) and also evades proofreading by viral exoribonuclease, resulting in decrease in viral RNA production [28,29]. It is a pro-drug, converted to ribonucleotide analogue GS-441524 in the body. The affected countries have resorted to social distancing by implementing lockdowns to check human to human spread of the virus, and also community spread (stage 3). 
Immune response and protection

Immune protection against invading pathogen/microbe(s) comprises of both innate and adaptive immunity. Innate immunity recruits immune cells to the site of infection through production of chemical factors called cytokines, activates complement cascade and adaptive immune system through antigen presentation. Inflammation, a protective response involving immune cells, molecular mediators and blood vessels, is one of the first responses of the immune system to infection initiated by cells present in all the tissues, viz., macrophages, dendritic cells, histiocytes, Kupffer cells, and mast cells.

The major histocompatibility complex (MHC) is a large locus on vertebrate DNA containing a set of closely linked polymorphic genes that code for cell surface proteins essential for the adaptive immune system. In humans, the MHC gene cluster is divided into three regions: classes I, II, and III. MHC molecules (also known as histocompatibility antigens) are highly polymorphic glycoproteins encoded by MHC class I and MHC class II genes, which are involved in the presentation of peptide antigens to T cells. Human MHC class I and II molecules are also called human leukocyte antigen (HLA). The MHC molecules bind peptide fragments from pathogens and display them on the cell surface for recognition by the appropriate $\mathrm{T}$ cells, that results in killing of virus infected cells. Besides, Blymphocytes are sensitized to produce antibodies to neutralize the pathogen(s). MHC class I molecules are expressed on all nucleated cells and present antigens/immunological domains (epitopes) to killer T cells (cytotoxic T lymphocytes; CTLs), and help in eliciting cellular immune response to counter intracellular pathogens, such as viruses and other intracellular microbes. MHC class II molecules occurs mostly on antigen-processing/presenting cells (APCs), including macrophages, and dendritic cells.MHC Class III molecules include several secreted proteins with immune functions, viz., components of the complement system, cytokines like TNF- $\alpha$, and heat shock proteins_[30]. Not much is known about the process of immune-response to the SARS-CoV-2.

Immuno-protective response to viral infections include activation of both innate and specific (adaptive) immune systems of the body. Viral infections usually activate cellular immune response involving both $\mathrm{CD}^{+}{ }^{+} \mathrm{T}$-lymphocytes (helper T cells, Th) and CD8 ${ }^{+}$ T-lymphocytes (cytotoxic T cells, CTLs). The CD4 Th lymphocytes help CTLs and B- lymphocytes that produce specific anti-viral antibodies [31]. The CD8 CTLs secrete a large number of biomolecules including Interferons (ability to interfere with virus proliferation) and perforin (pore forming cytolytic protein in the granules of
CTLs and natural killer (NK) cells) to eliminate viruses and virus (infected) altered cells from the infected/diseased host [32].

\section{Innate and adaptive immunity in SARS-CoV-2 infection}

Epithelial cells, alveolar macrophages and dendritic cells are three main components for innate immunity in the lung's airway [33]. Dendritic cells reside underneath the epithelium, whereas macrophages are located at the apical side of the epithelium. These two types of cells serve as innate immune cells to fight against viruses till adaptive immunity comes into play. T-Lymphocytes, both $\mathrm{CD}^{+}$and $\mathrm{CD}^{+}$, play important role in anti-viral immune response. There has been no detailed study on immunity to SARS-CoV-2 in human. But studies on other coronaviruses contribute to believe that convalescence from COVID-19 may impart protection from SARS-CoV-2 re-infection.

\section{Patho-immunobiology of COVID-19}

COVID-19 patients with severe disease (including ARDS) have lymphopenia, particularly the reduction in peripheral blood $\mathrm{T}$ cells [11,18,34]. In a study [5] conducted on COVID-19 patients revealed high WBC count with significant reduction in the numbers of circulating lymphocytes (lymphocytopenia) and platelets, CD4:CD8 ratio of 2:1 as in the healthy individuals, and higher expression of CD8 and hyperactivation of CTLs. There was no significant change in the expression level of CD4 compared to normal individuals. This study [5] inferred that anti-viral immune response to SARS-CoV-2 infection is through over expression of CD8 and hyper activation of CTLs.

The perspectives on immune responses to COVID-19 has been elaborated by Shi., et al. (2020) [3]. During the incubation period and non-severe clinical stage, specific immune response is required to eliminate the virus and check progression to severe stage, that is ARDS. When and if, adaptive immune response fails or is inadequate, the virus propagates causing massive destruction of host cells that have high expression of ACE2 receptor, including alveolar cells, intestine (enterocytes) and kidney. Damaged cells induce inflammation in the lungs mediated by macrophages and granulocytes. Granulocytes (neutrophils, eosinophils, and basophils) and mast cells are important mediators of inflammatory response.

T-cell antigen receptors (paratopes) on $\mathrm{CD}^{+}$and $\mathrm{CD}^{+}$cells recognise antigens presented by antigen processing/ presenting cells (APCs). Genetic differences can contribute to individual variation in immune response. It could be advantageous to have HLA molecules with increased binding specificities to the SARS-CoV-2 antigens presented by antigen processing/ presenting cells [3]. Therefore, it 
is required to identify whether specific HLA loci and alleles (class I or II) are associated with development of immune protection to SARS-CoV-2.

T-cell mediated responses against coronaviruses have been studied. These responses are initiated by antigen(s)/ epitope(s) presented on dendritic cells (DCs) and macrophages. These cells can phagocytize virus infected cells [35] and then present antigen(s)/ epitope(s) to T cells. Sometimes, these cells can also get infected. Based on the Immunological Genome database (http://rstats.immgen.org), there is limited expression of ACE2 on splenic dendritic cells and alveolar macrophages. SARS-CoV (of 2003) can bind to dendritic-cell specific intercellular adhesion molecule-3-grabbing nonintegrin (DC-SIGN) and DC-SIGN-related protein (DC-SIGNR, L-SIGN) in addition to ACE2 [36]. DC-SIGN is highly expressed on dendritic cells and macrophages. This aspect in SARS-CoV-2 needs further investigation. The APCs move to the draining lymph nodes and present viral antigens/epitopes to CD $4^{+}$ and $\mathrm{CD}^{+} \mathrm{T}$-lymphocytes, the former activate B- lymphocytes for the production of virus-specific antibody, while the latter functions as cytotoxic T-lymphocytes/cells (CTLs) and kill virus infected cells.

\section{Role of cytokines}

Patients with severe Cov-2 infection have been reported to have increased plasma concentrations of proinflammatory cytokines, including interleukin (IL)-6, IL-10, granulocyte-colony stimulating factor (G-CSF), monocyte chemoattractant protein 1 (MCP1), macrophage inflammatory protein (MIP) $1 \alpha$ and tumour necrosis factor $\alpha(\mathrm{TNF} \alpha)[19,34,37]$. The more severe condition was associated with higher level of IL-6. Patients having activated CD4 ${ }^{+}$ and $\mathrm{CD}^{+} \mathrm{T}$ cells had higher expression of CD69, CD38 and CD44 markers. NK group 2 member A (NKG2A), another marker for exhaustion was elevated on $\mathrm{CD}^{+} \mathrm{T}$ cells [38]. Exhaustion of $\mathrm{T}$ cells might lead to the progression of the disease. Another finding was that in COVID-19 patients with severe disease, there were aberrant pathogenic $\mathrm{CD}^{+} \mathrm{T}$ cells co-expressing interferon- $\gamma$ (IFN- $\gamma$ ) and granulocyte-macrophage colony-stimulating factor (GM-CSF) [19]. GM-CSF production by $\mathrm{T}$ cells has been previously reported as a response to virus infection. GM-CSF can help to differentiate innate immune cells and augment T cell function; but when in excess, it can initiate tissue damage [39]. GM-CSF+IFN- $\gamma+\mathrm{CD} 4^{+}$T cells were seen having strong $\mathrm{T}$ cell receptor (TCR) response in experimental autoimmune encephalomyelitis models, where $\mathrm{CD}^{+} \mathrm{T}$ cells expressing GM-CSF were found at higher percentage and secreted
IL-6. These immunological studies were exclusively reported from adult patients. Immunological responses in paediatric population needs to be examined.

The study of SARS-CoV of 2003 had revealed that virus infected lung epithelial cells produce IL-8 in addition to IL-6 [33]. IL-8 is a chemoattractant for neutrophils and T cells. Infiltration of a large number of inflammatory cells were observed in the lungs of severe COVID-19 patients [40-42] and these cells presumably consisted of innate immune cells and adaptive immune cells. Among innate immune cells, there could be abundance of neutrophils that can induce lung injury [43]. The majority of the infiltrating adaptive immune cells could be $\mathrm{T}$ cells, as there was significant reduction in circulating $\mathrm{T}$ cells. The $\mathrm{CD}^{+} \mathrm{T}$ cells are primary cytotoxic $\mathrm{T}$ cells. Severely sick patients also had cytotoxic $\mathrm{T}$ cells derived from $\mathrm{CD} 4^{+}$ $\mathrm{T}$ cells [44]. These cytotoxic T cells can kill virus and contribute to lung injury. Circulating monocytes respond to GM-CSF released by these pathological $\mathrm{T}$ cells. $\mathrm{CD} 14^{+} \mathrm{CD} 16^{+}$inflammatory monocytes, which rarely existed in healthy controls, were also found at significantly higher percentage in COVID-19 patients. These inflammatory $\mathrm{CD} 14^{+} \mathrm{CD} 16^{+}$monocytes had high expression of IL-6, which likely accelerated the progression of systemic inflammatory response. Another observation was that ACE2 was significantly expressed on innate lymphoid cells (ILC2 and ILC3). NK cells are a member of ILC1, which constitute a large portion of ILCs in the lung ( 95\%). ILC2 and ILC3 contribute to mucous homeostasis. There is a very limited study of ILC2 and ILC3 in coronavirus infection. Innate lymphoid cells (ILCs) are innate immune cells, and in response to pathogenic tissue damage contribute to immunity by secreting signalling molecules (cytokines).

Endothelial cells also express ACE2 [45] and represent one third of the lung [46]. Microvascular permeability due to endothelial injury can facilitate viral invasion. The function of the endothelium includes promotion of vasodilation, fibrinolysis and anti-aggregation. As endothelium plays a significant role in regulation of thrombosis, hypercoagulable profiles seen in severe diseases may indicate endothelial injury.

It has been reported that ACE2 is abundantly expressed on well-differentiated ciliated epithelial cells [47]. Human lung and epithelial cells continue to develop following the birth. Therefore, ACE2 expression may be lower in paediatric population. From the lung gene expression analysis portal (https://research.cchmc. org/pbge/lunggens/mainportal.html), ACE2 expression in mice 
increased around at birth. Its expression reduced till around P10 (postnatal 10), then increased.

Effect of gender, age, immune response and cytokine storm on COVID-19

Gender may also affect ACE2 expression. ACE2 gene is located on the X-chromosome. Circulating level of ACE2 is higher in men than in women [48] and this may attribute for the difference in severity and mortality between men and women both in the adult and the paediatric population (birth to 16 years of age) [49,50].

Children have a different response to the SARS-CoV-2 than adults. With increase in age, continuous antigen stimulation and thymic involution leads to a shift in $\mathrm{T}$ cell subset distribution from naïve $\mathrm{T}$ cells to central memory $\mathrm{T}$ cells, effector $\mathrm{T}$ cells and effector memory $\mathrm{T}$ cells [51]. The process of ageing is accompanied by the loss of expression of co-stimulatory molecules such as CD27 and CD28, with increased susceptibility to infections [52]. CD27 is a member of the tumour necrosis factor receptor superfamily. CD28 is expressed on T cells and helps in T cell activation and survival. $\mathrm{T}$ cell stimulation through $\mathrm{CD} 28$ provides signal for the production of IL- 6 . At the early stage after birth, CD4+ $\mathrm{T}$ cells lack in production of Th1 (cellular immune system) associated proinflammatory cytokines and diverted toward Th2 (humoral immune system). $\mathrm{CD}^{+} \mathrm{T}$ cells also have reduced expression of cytotoxic and inflammatory mediators [53]. As studied in the experimental models, low killing ability of $\mathrm{T}$ cells at early stage after birth possibly explains susceptibility of infants to SARS-CoV-2 [54]. Severe COVID-19 infection is characterized by a massive proinflammatory response or cytokine storm [2,3] that results in ARDS and multiorgan dysfunction. Also, it has been observed that inflammatory responses in adults and children are different [55]. Ageing is associated with increasing proinflammatory cytokines that govern neutrophil functions and have been correlated with the severity of ARDS. Presence of other viruses in lungs and airways, common in young children, can let SARS-CoV-2 virus compete with them and limit its growth [56]. Comprehensive understanding of why children in general are less susceptible to severe COVID-19 would help to design immunotherapy to eradicate this virus. So far there is no animal model for SARS-CoV-2.

The symptoms due to SARS-CoV-2 infection, varies from minimal sickness to ARDS, as in SARS-CoV-1 (SARS-2003). The ARDS developed following SARS-CoV-2 infection has been named as cytokine release syndrome (CRS), and the cytokine pathway can be targeted for chemotherapy [2]. The cytokine storm in ARDS is reflected by release of large amounts of pro-inflammatory cyto- kines vi., INF $\alpha$, IFN $\gamma$, IL-1 $\beta$, IL-6, IL-12, IL-18, IL-33, TNF $\alpha$, TGF $\beta$ ) and chemokines (CCL2, CCL3, CCL5, CXCL8, CXCL9, CXCL10 etc) by immune effector cells [57]. The possible therapeutics targeting cellular receptors ACE2 and TMPRSS2 could be helpful in preventing early phases of the infection, but ARDS that is developed late in the infection process/cycle as a result of cytokine storms, in which immune and nonimmune cells release large amounts of proinflammatory cytokines, viz., IL-6, TNF $\alpha$ and chemokines causing severe damage to the host, is lethal [2]. This cytokine storm is facilitated by activation of both NF-kB (nuclear factor kappa B) and STAT3 (signal transducer and activator of transcription 3) upon infection of the respiratory system by the SARS-CoV-2. Activation of the NF-KB and STAT3 can activate IL-6 amplifier that induces various pro-inflammatory cytokines and chemokines, including IL-6. While considering the cytokine and chemokine network in Cov-2 infection, it is intriguing to note that although diffuse alveolar damage (DAD) as seen in ARDS has been reported in many post-mortem cases [14], other modes of damage including microvascular injury and thrombosis also appear to be a hallmark of many patients resulting in death [58].

Neutralizing domains/epitopes of SARS-CoV and SARS-CoV-2 are located in both receptor binding domain (RBD) and receptor binding motif with in the RBD [59]. In SARS-CoV, attachment of the virus to ACE2 receptor can be blocked by specific neutralizing antibodies that recognize RBD in the Spike(S) glycoprotein, and $\mathrm{S}$ mediated viral fusion with cell membrane can be blocked by neutralizing antibodies against the heptad repeat 2 (HR2) domain [60]. Neutralizing antibodies can also interact with complement, phagocytes and natural killer cells to clear the pathogen. Therefore, neutralizing antibodies, as well as non-neutralizing antibodies, can bind to the Fc receptors present on different immune cells, viz., macrophages, monocytes and B-lymphocytes and result in antibody dependent enhancement (ADE), as in SARS, that occurs when there is suboptimal antibody response [4]. The ADE upregulates pro-inflammatory cytokines and down regulate anti-inflammatory cytokines, and the danger of ADE is also possible in SARS-CoV-2 infection and this can complicate the pathology of COVID-19. While viral infections are generally considered to confer immunity, reinfection with Covid-2 have been reported and such infections may be more serious than the first exposure, a phenomenon also seen in Dengue virus infections. ADE was observed earlier in cats administered with experimental FIPV (feline infectious peritonitis virus; an alpha coronavirus) vaccine causing more severe disease (in immunized cats) than in control cats after live virus challenge [61]. As scientists attempt to test over 90 vaccine candidates around the 
world, safety evaluation should include consideration of ADE and similar reactions [4].

\section{Conclusion}

The last Global pandemic of Spanish Flue due to Influenza A virus H1N1 occurred in 1918. People of all ages and sexes were affected with the disease. Since January 2020, the SARS-CoV-2 infection causing COVID-19 has become a global pandemic affecting 212 countries and territories covering all the five continents. As no proven vaccine or specific chemotherapy is currently available for COVID-19, the control of the spread of virus infection is being managed worldwide by adopting social distancing to prevent person-to-person spread and community transmission. It has been observed that the COVID-19 leads to ARDS in elderly people $>60$ years of age with high fatality rate. Though, enough information is not available on the immunopathology of the disease, available data suggests that untoward immunological reactions (immunopathology) involving cellular immunity lead to 'cytokine storm' causing 'cytokine release syndrome' in patients having ARDS. Further, neutralizing antibodies as well as non-neutralizing antibodies can lead to ADE involving cells of immune system with Fc receptors. As on 04 May 2020 (05:02 GMT), there were >3.56 million cases worldwide with $7 \%$ mortality (CFR) and 1.154 million people recovered from the disease (www.worldometers.info/coronavirus). The pandemic has caused a severe blow to the economy and lifestyle in USA and Europe. In USA, the total number of cases is about 1.188 million as on 04 May 2020 (05:02 GMT) with CFR of 5.77\%, whereas, In India, with a population of about 1300 million (1.3 billion), there were only 42,533 cases with 1,391 deaths, and CFR of 3.27\%. In smaller European countries including Spain, Italy, U.K., France, and Belgium, the CFR was in the range of 10.22 to $15.7 \%$. In Russia and Germany, the CFR was much lesser at $0.95 \%$ and $4.14 \%$, respectively. It is possible that there is genetic susceptibility/resistance to COVID-19. It has been recorded elsewhere that all exposed to the virus do not get infected. As per Shi., et al. (2020), it is important to identify whether there are any specific HLA loci and alleles (either class I or II) associated with SARS-CoV-2 protective immunity. In addition, it will be of interest to investigate whether the countries like India where BCG (Bacillus CalmetteGuerin) vaccination at birth (for prevention against tuberculosis and leprosy) is in vogue since many years, have stronger innate immunity in the population that may resist community spread of the virus infection. In countries where tuberculosis or leprosy is common, one dose of BCG vaccine is administered to babies as close to the time of birth as possible. Different species of Mycobacterium genus including M. phlei are known to excite stronger innate immunity that continues for long period in life. Further, it remains to be seen whether after the COVID-19 pandemic slows down (post pandemic period), virus persistence and carrier state is established and continues in the population. In the present scenario of the pandemic, there is urgent need for preparedness in terms of quick and precise diagnostics to monitor presence of viral antigen or antibodies in human population under structured studies. In addition, circulation of SARS-CoV-2 needs to be monitored in different animal species, like pets (dogs and cats) which remain in close contact with human beings, and possibility of reverse zoonoses. The veterinarians, animal virologists, pathologists and immunologists have a greater role to play towards the control, prevention and management of COVID-19 and similar pandemics of zoonotic nature in future along with medical professionals, molecular biologists and epidemiologists under 'one health' concept.

\section{Acknowledgement}

Information available in the Internet (NCBI-PubMed, WHO, OIE, ICMR, ICAR, Govt of India, Wikipedia, Newspaper and News periodicals etc) have been incorporated in the present review. We sincerely acknowledge each of these.

\section{Bibliography}

1. Hoffmann M., et al. "SARS-CoV-2 cell entry depends on ACE2 and TMPRSS 2 and is blocked by a clinically proven protease inhibitor". Cell 181 (2020): 271-280.

2. Hirano T and Murakami M. "COVID-19: A new virus, but a familiar receptor and cytokine release syndrome". Immunity 52.5 (2020): 731-733.

3. Shi Y., et al. "COVID-19 infection: the perspectives on immune responses". Cell Death and Differentiation 27 (2020): 14511454.

4. Iwasaki A and Yang Y. The potential danger of suboptimal antibody response in COVID-19.

5. Ganji A., et al. "Increased expression of CD8 marker on T-cells in COVID-19 patients". Blood Cells Molecules and Diseases 83 (2020): 102437.

6. Yuki K., et al. "COVID-19 pathophysiology: A review". Clinical Immunology (2019): 108427.

7. Sahu K K., et al. "COVID-19: update on epidemiology, disease spread and management". Monaldi Archives Chest Disease 90.1292 (2020): 197-205. 
8. Bar-On YM., et al. "SARS-CoV-2 (COVID-19) by numbers". eLife (2020): 57309.

9. Lu R., et al. Genomic characterization and epidemiology of 2019 novel coronavirus: implications for virus origins and receptor binding". The Lancet 395 (2020): 565-574.

10. Wang D., et al. "Clinical characteristics of 138 hospitalized patients with 2019 novel coronavirus infected pneumonia in Wuhan, China". The Journal of the American Medical Association 323.11 (2020): 1061-1069.

11. Zhou F., et al. "Clinical course and risk factors for mortality of adult inpatients with COVID-19 in Wuhan, China: a retrospective cohort study". Lancet 395(10229):1054-1062.

12. Gautret P., et al. "Hydroxychloroquine and azithromycin as a treatment of COVID-19: results of an open-label non-randomized clinical trial". The International Journal of Antimicrobial Agents (2020): 105949.

13. Rabi F A., et al. "SARS-CoV-2 and Coronavirus Disease 2019: What We Know So Far". Pathogens 9.3 (2020): 231.

14. Xiao K., et al. "Isolation of SARS-CoV-2-related coronavirus from Malayan pangolins". Nature (2020).

15. Wu F., et al. "A new coronavirus associated with human respiratory disease in China". Nature 579.7798 (2020): 265-269.

16. Chen Y., et al. "Structure analysis of the receptor binding of 2019-nCoV". Biochemical and Biophysical Research Communications 525.1 (2020): 135-140.

17. Li W., et al. "Angiotensin-converting enzyme 2 is a functional receptor for the SARS coronavirus". Nature (2003).

18. Zhou P., et al. "A pneumonia outbreak associated with a new coronavirus of probable bat origin". Nature 579.7789 (2020):270-273.

19. Zhou Y., et al. "Pathogenic T cells and inflammatory monocytes incite inflammatory storm in severe COVID-19 patients". National Science Review (2020).

20. Walls A C., et al. "Structure, Function, and Antigenicity of the SARS-CoV-2 Spike Glycoprotein". Cell 181.2 (2020): 281-292.
21. Zou X., et al. "Single-cell RNA-seq data analysis on the receptor ACE2 expression reveals the potential risk of different human organs vulnerable to 2019-nCoV infection". Frontiers in Medicine 14.2 (2020):185-192.

22. Ou X., et al. "Characterization of spike glycoprotein of SARSCoV2 on virus entry and its immune cross-reactivity with SARS-CoV". Nature Communications 11.1 (2020): 1620.

23. Belouzard S., et al. "Mechanisms of coronavirus cell entry mediated by the viral spike protein". Viruses 4.6 (2012): 10111033.

24. Hamming I., et al. "Tissue distribution of ACE2 protein, the functional receptor for SARS coronavirus. A first step in understanding SARS pathogenesis". The Journal of Pathology 203.2 (2004): 631-637.

25. Chen N., et al. "Epidemiological and clinical characteristics of 99 cases of 2019 novel coronavirus pneumonia in Wuhan, China: a descriptive study". Lancet 395.10223 (2020): 507-513.

26. Wu Z and McGoogan J M. "Characteristics of and important lessons from the Coronavirus Disease 2019 (COVID-19) outbreak in China: Summary of a report of 72314 cases from the chinese center for disease control and prevention". The Journal of the American Medical Association (2020).

27. Onder G., et al. "Case-Fatality Rate and Characteristics of Patients Dying in Relation to COVID-19 in Italy". The Journal of the American Medical Association (2020).

28. Ferner R E and Aronson J K. "Remdesivir in covid-19". British Medical Journal 369 (2020): m1610.

29. Scavone C., et al. "Current pharmacological treatments for COVID-19: what's next?" British Journal of Pharmacology (2020).

30. Janeway C A Jr., et al. "Immunobiology: The Immune System in Health and Disease". $5^{\text {th }}$ edition. New York: Garland Science (2001).

31. Zhu J., et al. "Differentiation of effector CD4 T cell populations". The Annual Review of Immunology 28 (2009): 445-489.

32. Mescher M F., et al. "Signals required for programming effector and memory development by CD8+ T cells". Immunological Reviews 211 (2006): 81-92. 
33. Yoshikawa T., et al. "Severe acute respiratory syndrome (SARS) coronavirus-induced lung epithelial cytokines exacerbate SARS pathogenesis by modulating intrinsic functions of monocyte-derived macrophages and dendritic cells". Journal of Virology 83.7 (2009): 3039-3048.

34. Qin C., et al. "Dysregulation of immune response in patients with COVID-19 in Wuhan, China". Clinical Infectious Diseases 12 (2020): ciaa248.

35. Fujimoto I., et al. "Virus clearance through apoptosis dependent phagocytosis of influenza A virus-infected cells by macrophages". Journal of Virology 74.7 (2000): 3399-3403.

36. Yang Z Y., et al. "pH-dependent entry of severe acute respiratory syndrome coronavirus is mediated by the spike glycoprotein and enhanced by dendritic cell transfer through DCSIGN". Journal of Virology 78.11 (2004): 5642-5650.

37. Huang C., et al. "Clinical features of patients infected with 2019 novel coronavirus in Wuhan, China”. Lancet 395.10223 (2020): 497-506.

38. Zheng M., et al. "Functional exhaustion of antiviral lymphocytes in COVID-19 patients". Cellular and Molecular Immunology 17.5 (2020): 533-535.

39. Huang H., et al. "High levels of circulating GM-CSF (+) CD4(+) $\mathrm{T}$ cells are predictive of poor outcomes in sepsis patients: a prospective cohort study". Cellular and Molecular Immunology 16.6 (2019): 602-610.

40. Xu Z., et al. "Pathological findings of COVID-19 associated with acute respiratory distress syndrome". The Lancet Respiratory Medicine 8.4 (2020): 420-422.

41. Tian S., et al. "Pulmonary Pathology of Early-Phase 2019 Novel Coronavirus (COVID-19) Pneumonia in Two Patients with Lung Cancer". Journal of Thoracic Oncology 15.5 (2019): 700704.

42. Tian S., et al. "Pathological Study of the 2019 Novel Coronavirus Disease (COVID-19) Through Postmortem Core Biopsies". Modern Pathology (2020b): 1-8.

43. Koutsogiannaki S., et al. "The Use of Volatile Anesthetics as Sedatives for Acute Respiratory Distress Syndrome". Translational Perioperative and Pain Medicine 6.2 (2019): 27-38.
44. Fang M., et al. "Perforin-dependent CD4+ T-cell cytotoxicity contributes to control a murine poxvirus infection". Proceedings of the National Academy of Sciences of the United States of America 109.25 (2012): 9983-9988.

45. Lovren F., et al. "Angiotensin converting enzyme-2 confers endothelial protection and attenuates atherosclerosis". The American Journal of Physiology-Heart and Circulatory Physiology 295 (2008): H1377-H1384.

46. Zeng H., et al. "Human pulmonary microvascular endothelial cells support productive replication of highly pathogenic avian influenza viruses: possible involvement in the pathogenesis of human H5N1 virus infection". Journal of Virology 86.2 (2012): 667-678.

47. Jia H P., et al. "ACE2 receptor expression and severe acute respiratory syndrome coronavirus infection depend on differentiation of human airway epithelia". Journal of Virology 79.23 (2005): 14614-14621.

48. Patel S K., et al. "Emerging markers in cardiovascular disease: where does angiotensin-converting enzyme 2 fit in?" Clinical and Experimental Pharmacology and Physiology 40.8 (2013): 551-559.

49. Wenham C., et al. "COVID-19: the gendered impacts of the outbreak". Lancet 395.10227 (2020): 846-848.

50. Jin J., et al. "Gender differences in patients with COVID-19: Focus on severity and mortality". Frontiers in Public Health 8 (2020): 152.

51. Saule P., et al. "Accumulation of memory T cells from childhood to old age: central and effector memory cells in CD4(+) versus effector memory and terminally differentiated memory cells in CD8(+) compartment". Mechanisms of Ageing and Development 127.3 (2006): 274-281.

52. Li M., et al. "Age related human T cell subset evolution and senescence". Immunity and Ageing (2019).

53. Connors T J., et al. "Airway CD8(+) T Cells Are Associated with Lung Injury during Infant Viral Respiratory Tract Infection". The American Journal of Respiratory Cell and Molecular Biology 54 (2016): 822-830. 
54. Smits S L., et al. "Exacerbated innate host response to SARS$\mathrm{CoV}$ in aged non-human primates". PLOS Pathogens 6.2 (2010): e1000756.

55. Wong H R., et al. "Leukocyte subset derived genome wide expression profiles in pediatric septic shock". Pediatric Critical Care Medicine 11.3 (2010): 349-355.

56. Nickbakhsh S., et al. "Virus-virus interactions impact the population dynamics of influenza and the common cold". Proceedings of the National Academy of Sciences of the United States of America 116.52 (2019): 27142-27150.

57. Thuen Jan von der and Erden Menno van der. "Histopathology and genetic susceptibility in COVID-19 pneumonia". European Journal of Clinical Investigation (2020).

58. Margo C., et al. "Complement Associated Microvascular Injury and Thrombosis in the Pathogenesis of Severe COVID-19 Infection: A Report of Five Cases". Translational Research S19315244.20 (2020): 30070-30070.

59. Chen W-H., et al. "Potential for developing a SARS-CoV receptor binding domain (RBD) recombinant protein as a heterologous human vaccine against coronavirus infectious disease (COVID)-19". Human Vaccines and Immunotherapeutics (2020).

60. Du L., et al. "The spike protein of SARS-CoV- a target for vaccine and therapeutic development". Nature Reviews Microbiology 7 (2009): 226-236.

61. Decaro N., et al. "COVID-19 from veterinary medicine and one health perspectives: What animal coronaviruses have taught us". Research in Veterinary Science 131 (2020): 21-23.

\section{Assets from publication with us}

- Prompt Acknowledgement after receiving the article

- Thorough Double blinded peer review

- Rapid Publication

- Issue of Publication Certificate

- High visibility of your Published work

Website: https://www.actascientific.com/

Submit Article: https://www.actascientific.com/submission.php Email us: editor@actascientific.com

Contact us: +919182824667 